$12-14-2005$

\title{
Kondo Resonances and Anomalous Gate Dependence in the Electrical Conductivity of Single-Molecule Transistors
}

L H. Yu

Z K. Keane

Jacob W. Ciszek

Loyola University Chicago, jciszek@luc.edu

L Cheng

J M. Tour

See next page for additional authors

Follow this and additional works at: https://ecommons.luc.edu/chemistry_facpubs

Part of the Chemistry Commons

\section{Recommended Citation}

Yu, LH, ZK Keane, JW Ciszek, L Cheng, JM Tour, T Baruah, MR Pederson, and D Natelson. "Kondo resonances and anomalous gate dependence in the electrical conductivity of single-molecule transistors" in Physical Review Letters 95, 2005.

This Article is brought to you for free and open access by the Faculty Publications and Other Works by Department at Loyola eCommons. It has been accepted for inclusion in Chemistry: Faculty Publications and Other Works by an authorized administrator of Loyola eCommons. For more information, please contact ecommons@luc.edu. cc) (i) $\Theta$

This work is licensed under a Creative Commons Attribution-Noncommercial-No Derivative Works 3.0 License. (c) 2005 American Physical Society. 


\section{Authors}

L H. Yu, Z K. Keane, Jacob W. Ciszek, L Cheng, J M. Tour, T Baruah, M R. Pederson, and D Natelson 


\title{
Kondo Resonances and Anomalous Gate Dependence in the Electrical Conductivity of Single-Molecule Transistors
}

\author{
L. H. Yu, ${ }^{1}$ Z. K. Keane, ${ }^{1}$ J. W. Ciszek, ${ }^{2}$ L. Cheng, ${ }^{2}$ J. M. Tour, ${ }^{2}$ T. Baruah, ${ }^{4,5}$ M. R. Pederson, ${ }^{4}$ and D. Natelson ${ }^{1,3}$ \\ ${ }^{1}$ Department of Physics and Astronomy, Rice University, 6100 Main Street, Houston, Texas 77005, USA \\ ${ }^{2}$ Department of Chemistry and Center for Nanoscale Science and Technology, Rice University, \\ 6100 Main Street, Houston, Texas 77005, USA \\ ${ }^{3}$ Department of Electrical and Computer Engineering, Rice University, 6100 Main Street, Houston, Texas 77005, USA \\ ${ }^{4}$ Center for Computational Materials Science, Code 6390-Naval Research Laboratory, Washington, DC 20375-5435, USA \\ ${ }^{5}$ Department of Physics, SUNY, Stony Brook, New York 11794, USA
}

(Received 27 May 2005; published 14 December 2005)

\begin{abstract}
We report Kondo resonances in the conduction of single-molecule transistors based on transition metal coordination complexes. We find Kondo temperatures in excess of $50 \mathrm{~K}$, comparable to those in purely metallic systems. The observed gate dependence of the Kondo temperature is inconsistent with observations in semiconductor quantum dots and a simple single-dot-level model. We discuss possible explanations of this effect, in light of electronic structure calculations.
\end{abstract}

DOI: 10.1103/PhysRevLett.95.256803

In the Kondo Hamiltonian [1], one of the most wellstudied many-body problems in physics, an unpaired spin localized in a singly occupied electronic level, is coupled via tunneling to an electronic bath. On-site Coulomb repulsion forbids real double occupancy of the level, but virtual processes favor antiferromagnetic exchange between the local spin and the electronic bath. As $T$ is reduced below a characteristic Kondo temperature, $T_{K}$, these exchange processes "screen" the local moment. The Kondo problem has undergone a resurgence, with atomic-scale studies of Kondo physics by scanning tunneling microscopy (STM) [2,3], and the realization of tunable Kondo systems in semiconductor quantum dots [4-7]. With the recent development of single-molecule transistors (SMTs) based on individual small molecules [8], Kondo systems now include organometallic compounds $[9,10]$ and fullerenes with normal [11] and ferromagnetic [12] leads.

In this Letter, we report Kondo physics in SMTs incorporating transition metal complexes designed to contain unpaired electrons. As a function of gate voltage, $V_{G}$, we observe transitions from Coulomb blockade conduction to Kondo conduction, manifested as a strong peak in the differential conductance, $G \equiv \partial I_{\mathrm{D}} / \partial V_{\mathrm{SD}}$, at zero bias, $V_{\mathrm{SD}}=0$, in one charge state. At fixed $V_{G}$, the temperature dependence of the conductance peaks' amplitudes and widths agree well with the expected forms for spin-1/2 Kondo resonances. Observed SMT Kondo temperatures are $>\sim 50 \mathrm{~K}$, comparable to those in purely metallic Kondo systems. We find that $T_{\mathrm{K}}\left(V_{G}\right)$ is strongly inconsistent with the simple model of Kondo physics as seen in semiconductor devices [6,7]. We discuss explanations for this anomalous gate dependence in light of spin-resolved electronic structure calculations of the complexes.

Figure 1(a) shows the simple single-level picture often used to describe Kondo physics in single-electron transistors (SETs). The intrinsic width of the single-particle level
PACS numbers: 73.22.-f, 73.23.Hk, 85.65.+h

is $\Gamma \equiv \Gamma_{\mathrm{S}}+\Gamma_{\mathrm{D}}$, determined by overlap of the singleparticle state with the conduction electron states of the source and drain. The charging energy $E_{\mathrm{c}}$ is the Coulomb cost of adding an extra electron to the molecule. The energy difference between the singly occupied level and the source/drain chemical potential is $\epsilon$, which is zero at charge degeneracy and varies linearly with $V_{G}$.

Figure 1(b) shows the structure of the neutral transition metal complexes measured in this study, as synthesized. Two planar, conjugated ligands provide an octahedral coordination [compressed along the interligand $(z)$ axis] of a transition metal ion, $M$. We have examined complexes with $\mathrm{M}=\mathrm{Co}, \mathrm{Cu}$, and $\mathrm{Zn}$, as well as individual ligands and alkane chains. The Kondo devices in this Letter contain $\mathrm{Co}$ (II) and $\mathrm{Cu}$ (II). The as-synthesized molecules are characterized by EPR, SQUID, x-ray diffraction, cyclic vol-
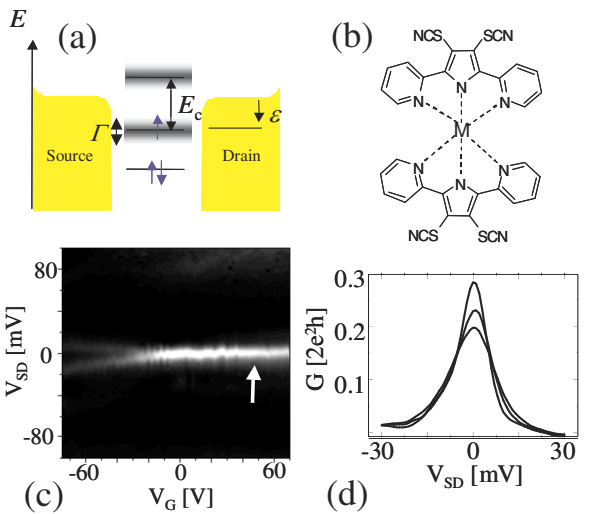

FIG. 1 (color online). (a) Energy level diagram of a typical SET in the Kondo regime. (b) Structural formula of transition metal complex before self-assembly. (c) Map of $G\left(V_{\mathrm{SD}}, V_{G}\right)$ showing transition to Kondo resonance at $5 \mathrm{~K}$. Brightness scales from $G=0$ (black) to $0.3 \times 2 e^{2} / h$. (d) $G$ vs $V_{\mathrm{SD}}$ at $V_{\mathrm{G}}=50 \mathrm{~V}$ [as indicated with arrow in (c)] at (top to bottom) $5 \mathrm{~K}, 16 \mathrm{~K}$, and $30 \mathrm{~K}$. 
tammetry (CV), IR, and Raman spectroscopy. The Co(II) complex is high spin (3/2), with CV in solution confirming easily accessible $\mathrm{Co}$ (II) $\leftrightarrow \mathrm{Co}$ (III) redox transitions. The $\mathrm{Cu}$ (II) complex is spin $1 / 2$, with $\mathrm{CV}$ supporting easily accessible $\mathrm{Cu}(\mathrm{II}) \leftrightarrow \mathrm{Cu}(\mathrm{I})$ redox transitions. These complexes self-assemble on $\mathrm{Au}$ in tetrahydrofuran (THF) through loss of the $-\mathrm{CN}$ moieties and formation of $\mathrm{Au}-\mathrm{S}$ covalent bonds [13-15]. In principle, the ligands and remaining $-\mathrm{SCN}$ groups can also change their charge state.

Devices are fabricated using Au source and drain electrodes on degenerately doped $p+$ silicon substrates which are used as the gate, with $200 \mathrm{~nm}$ of gate oxide. Source/ drain electrodes are created by the controlled electromigration [16] from lithographically defined $\mathrm{Ti} / \mathrm{Au}$ $(1 \mathrm{~nm} / 15 \mathrm{~nm}$ thick) constrictions that are exposed for $1 \mathrm{~min}$ to oxygen plasma after lift-off, followed by selfassembly of molecules in solution ( $2 \mathrm{mM}$ in THF) for 48 hours. After rinsing and drying with dry nitrogen, substrates are placed in a variable temperature probe station for electromigration and measurement, with fabrication statistics comparable to past results $[11,14]$.

dc measurements of $I_{\mathrm{D}}-V_{\mathrm{SD}}$ are performed with the source electrode grounded, at various $V_{G}$. Differential conductance is computed by numerical differentiation, with spot checks by lock-in amplifier techniques. Device stability limits $\left|V_{\mathrm{SD}}\right| \leq 100 \mathrm{mV}$, while gate oxide limits $\left|V_{G}\right| \leq 100 \mathrm{~V}$. We consider only devices with significant gate response such that a charge transition is detected in maps of $G\left(V_{\mathrm{SD}}, V_{G}\right)$ to distinguish molecule-based effects from artifacts (e.g., metal nanoparticles).

Figure 1(c) shows a conductance map for a typical device exhibiting a Kondo resonance. We have sufficient gate coupling to observe only a single charge degeneracy point for each device. From the slopes of the boundaries of the blockaded regime, one can estimate the constant of proportionality between changes in $e V_{G}$ and $\epsilon$ as expected from the model of Fig. 1(a). For the device shown, $\delta \epsilon \approx$ $\left(C_{G} / C_{\text {tot }}\right) e \delta V_{G} \approx 10^{-4} e \delta V_{G}$. The average value of this coefficient is $10^{-3}$. The width of the charge degeneracy resonance on the blockaded side of the degeneracy point sets an upper limit on $\Gamma$ for the level participating in the redox state change. The $T \rightarrow 0$ limit of that width is proportional to $\Gamma$, while the degeneracy resonance is thermally broadened at finite temperature. Typical $\Gamma$ values inferred from $5 \mathrm{~K}$ data in these devices are 3-30 meV. We note that the edges of Coulomb blockade diamonds, while usually distinct in the non-Kondo charge state, are often much weaker or apparently absent in the Kondo charge state, as also mentioned in Ref. [12].

Figure 1(d) shows $G\left(V_{\mathrm{SD}}, V_{G}=50 \mathrm{~V}\right)$ at three temperatures. The resonant peak decreases in magnitude while increasing in width as $T$ is increased. Figures 2(a) and 2(b) show the peak height and width, respectively, as a function of temperature. The solid line in (a) is a fit to the semiempirical expression [4] for the spin- $1 / 2$ Kondo resonance in conduction, $G(T)=G_{\mathrm{c}}\left(1+2^{1 / s-1} \frac{T^{2}}{T_{\mathrm{K}}^{2}}\right)^{-s}$, with $s=0.22$. After the subtraction of a smooth background conductance, the adjustable parameters are the overall conductance scale, $G_{\mathrm{c}}$, and $T_{\mathrm{K}}$. Similarly, the solid line in (b) is a fit to the expected form [3] for the full width at half maximum, FWHM $=\frac{2}{e} \sqrt{\left(\pi k_{\mathrm{B}} T\right)^{2}+2\left(k_{\mathrm{B}} T_{\mathrm{K}}\right)^{2}}$, where the only adjustable parameter is $T_{\mathrm{K}}$. The numerical coefficients are accurate to within a factor of order unity; other observations [7,10] find better quantitative consistency with $T_{\mathrm{K}}$ as inferred from $G\left(V_{\mathrm{SD}}=0, T\right)$ if the FWHM is assumed to be $\approx\left(2 k_{\mathrm{B}} T_{\mathrm{K}}\right) / e$. We adopt this latter assumption, and find good quantitative consistency between $T_{\mathrm{K}}$ values inferred from resonance height and width.

Figures 2(c) and 2(d) are histograms of Kondo temperatures inferred from resonance widths for $26 \mathrm{Co}$-containing Kondo devices (out of 921 electrode pairs examined at low temperatures) and $12 \mathrm{Cu}$-containing Kondo devices (out of 397 electrode pairs examined at low temperatures). Only devices exhibiting zero-bias resonances and clear charge degeneracy points are considered here. The observed weak dependence of $T_{\mathrm{K}}$ on $V_{G}$ (see Fig. 3 and later discussion) means that these distributions are relatively insensitive to the choice of $V_{G}$ at which $T_{\mathrm{K}}$ is inferred. No Kondo resonances were observed in 370 control devices using alkanethiol chains, bare metal electrodes, and electrodes exposed to solvents and poor vacuum.

Kondo physics in these complexes is clearly strong, with $T_{\mathrm{K}}$ values similar to those reported in STM measurements of $\mathrm{Co}$ atoms on $\mathrm{Au}(111)$ [2]. The Kondo temperature is expected to be $[7,17]$ :

$$
k_{\mathrm{B}} T_{\mathrm{K}}=\frac{\sqrt{\Gamma E_{\mathrm{c}}}}{2} e^{-\pi \epsilon\left(-\epsilon+E_{\mathrm{c}}\right) / \Gamma E_{\mathrm{c}}}
$$

outside the mixed valence $(\epsilon / \Gamma<1)$ regime. For small molecules, $E_{\mathrm{c}}$ is likely to be hundreds of $\mathrm{meV}$, while $\Gamma$ is empirically tens of meV. The prefactor in (1) then implies $T_{\mathrm{K}}$ can be as large as hundreds of Kelvin.

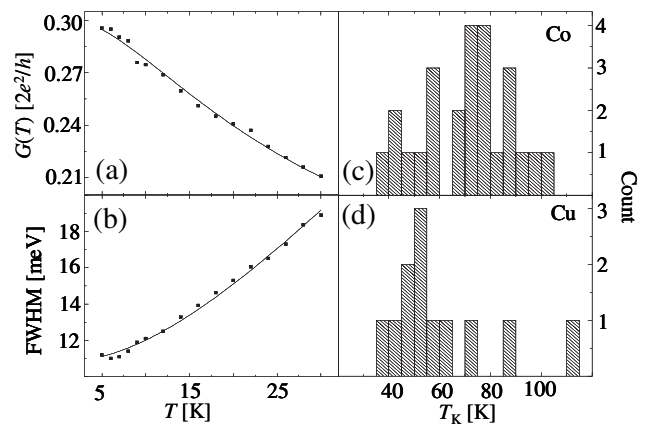

FIG. 2. (a) Temperature dependence of Kondo resonant peak height for the device of Fig. 1(c) at $V_{G}=50 \mathrm{~V}$. Solid line is the expected semiempirical functional form for spin-1/2 Kondo, with $T_{\mathrm{K}}=69 \mathrm{~K}$. (b) Temperature dependence of Kondo peak FWHM for the same device and $V_{G}$, with fit to expected functional form for spin-1/2 Kondo. Setting FWHM in the low- $T$ limit to $2 k_{\mathrm{B}} T_{\mathrm{K}} / e$ gives $T_{\mathrm{K}}=65 \mathrm{~K}$. (c), (d) Histograms of $T_{\mathrm{K}}$ as inferred from peak widths for $\mathrm{Co}$ and $\mathrm{Cu}$ complex devices, respectively. 


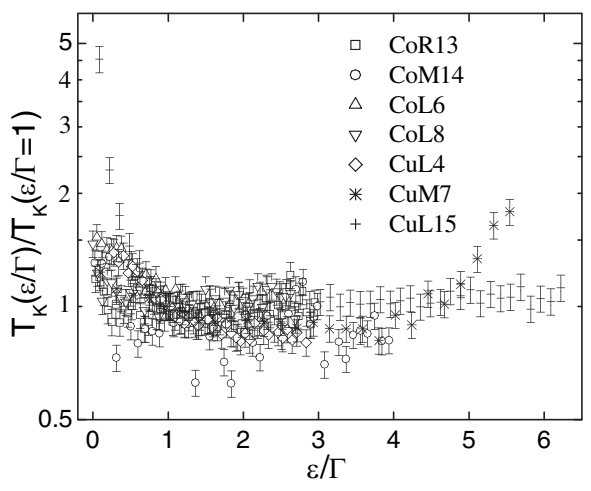

FIG. 3. $\log T_{\mathrm{K}}$ (normalized) inferred from FWHM of $5 \mathrm{~K}$ Kondo peak in $G\left(V_{\mathrm{SD}}\right)$, as a function of $V_{G}$ normalized by the width of the Coulomb blockade charge degeneracy point $[=$ $\epsilon / \Gamma$ in the simple model of Fig. 1(a)] for several devices. In the model of Fig. 1(a), a parabolic dependence of $\log T_{\mathrm{K}}$ on $V_{G}$ is expected. Values of $\Gamma$ inferred for these devices are, top down, $22.6,11.5,18,3.3,12.6,14.2$, and $26.8 \mathrm{meV}$.

Assuming a model as in Fig. 1(a), $\epsilon=0$ at the charge degeneracy point. Normalizing the difference in $V_{G}$ away from charge degeneracy by the width in $V_{G}$ of the charge degeneracy resonance in the Coulomb blockade regime gives a lower limit on $\epsilon / \Gamma$. Figure 3 shows (normalized) $T_{\mathrm{K}}$ as inferred from low- $T$ resonance FWHM $\sim 2 k_{\mathrm{B}} T_{\mathrm{K}} / e$ as a function of inferred $\epsilon / \Gamma$ for several devices. Equation (1) predicts a quadratic dependence of $\log T_{\mathrm{K}}$ on $\epsilon$, with a minimum in $T_{\mathrm{K}}$ at $\epsilon=E_{\mathrm{c}} / 2$. The measured dependence of inferred $T_{\mathrm{K}}\left(V_{G}\right)$ is much less steep; indeed, for Sample CuM7, $T_{\mathrm{K}}$ actually increases as $V_{G}$ is shifted away from the nearest charge degeneracy. Thus, the simple model of Fig. 1(a), which works well for semiconductor quantum dot experiments [6,7], is inconsistent with these data. We note that the $T_{\mathrm{K}}\left(V_{G}\right)$ dependence reported in Ref. [9] for $\epsilon / \Gamma>1$ is also surprisingly weak.

We consider explanations for this deviation from simple expectations. We dismiss as unlikely that the zero-bias peak may not be a true Kondo resonance, given (a) quantitative consistency of the functional forms for the resonance $G\left(V_{\mathrm{SD}}, T\right)$ with Kondo expectations; (b) the appearance of the zero-bias resonance coincident with passing through charge degeneracy points; (c) the lack of such resonances in control devices; (d) the similarity to other Kondo data reported in SMTs $[9,10]$.

The natural explanation for the anomalous gate dependence is that the normalized $\delta V_{G}$ used as $\epsilon / \Gamma$ in Fig. 3 is not the true $\epsilon / \Gamma$ relevant to the Kondo Hamiltonian that gives Eq. (1). In semiconductor quantum dots [6], inferring $\epsilon / \Gamma$ by normalizing $\delta V_{G}$ is quantitatively consistent with Eq. (1). Presumably some mechanism intrinsic to the molecular system renormalizes either the effective $\epsilon$, the effective $\Gamma$, or both, away from the simple picture of Fig. 1(a).

Orbital degeneracy for the unpaired spin is one possibility [18]. When an $N$-fold degeneracy exists, $T_{K}$ as defined by Eq. (1) is enhanced, such that the denominator of the exponent becomes $N \Gamma E_{\mathrm{c}}$. Thus the normalization of the abcissa in Fig. 3 would effectively be too large by a factor of $N$, qualitatively explaining the apparently weak dependence of $T_{\mathrm{K}}\left(V_{G}\right)$ if $N \sim 5$. However, EPR spectra of both the $\mathrm{Co}-$ and $\mathrm{Cu}$-based complexes in solution phase show no indication of such a large degeneracy. Furthermore, we have performed spin-resolved calculations on the transition metal complexes to examine their electronic structure, as shown in Fig. 4 [further details are available [15] ]. In neither complex are large degeneracies expected. While it is conceivable that the self-assembled compounds could have different orbital degeneracies than the isolated molecules, it seems unlikely that both complexes, with their differing isolated electronic structures, would have such similar properties.

The electronic structure calculations reveal an additional energy scale that is often small in semiconductor dots, but in the molecular system is comparable energetically to the inferred $\Gamma, E_{\mathrm{c}}, \epsilon$, and expected single-particle level spacing: intramolecular exchange. In both $\mathrm{Co}$ and $\mathrm{Cu}$ complexes, intramolecular exchange is strong. In the Co case, to a good approximation ligand field effects split the $d$ states into one doubly degenerate, one nearly doubly degenerate, and one singly degenerate set of states. The complex has a total spin of $S=3 / 2$, as confirmed by EPR. A minority spin half-occupied $d$ state is at the Fermi level with two nearly degenerate $d$ states $1.1 \mathrm{eV}$ above the Fermi level and the remaining occupied minority $d$ electron $0.5 \mathrm{eV}$ below the Fermi level. Exchange splittings pull the majority $d$ band down significantly, with the highest occupied majority $d$ state $1.8 \mathrm{eV}$ below the Fermi level. The fermiology is complicated by an additional delocalized unpolarized twofold degenerate carbon $2 p$ state lying $0.05 \mathrm{eV}$ below the Fermi level. Also there is a delocalized doubly degenerate molecular state $0.85 \mathrm{eV}$ below the Fermi level. The $\mathrm{Cu}$ complex has a total spin of $S=1 / 2$, with the Fermi level unchanged in comparison

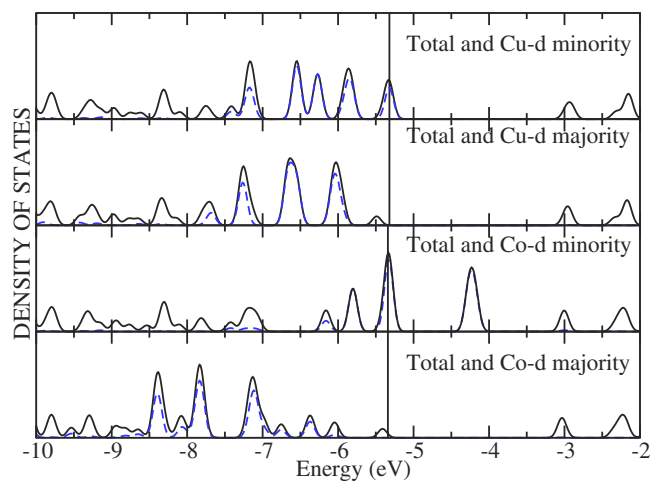

FIG. 4 (color online). Spin-resolved projected densities of states of the $\mathrm{Cu}$ (II) and $\mathrm{Co}(\mathrm{II})$ complexes, respectively. Minority (upper) and majority (lower) spin levels are indicated, demonstrating the strong effects of intramolecular exchange. The black curve is the total DOS, and the dashed curve (blue) is the DOS projected onto the transition metal $d$ states. Further details are available [15]. 
to Co. This means that the minority spin $d$ states are pulled to lower energies by approximately $1.1 \mathrm{eV}$ as shown in the figure. The structure calculations also confirm significant delocalization of the majority spin states, extending into the ligands, consistent with large $\Gamma$ values for these systems. Jahn-Teller distortion is not considered, since the substrate-complex interaction is expected to be large compared to this effect.

The occasional observation of Kondo resonances with similar $T_{\mathrm{K}}$ values on both sides of charge degeneracy points further indicates that intramolecular exchange cannot be neglected in these devices. However, it is not immediately clear how this strong interaction could lead to the observed phenomenon.

Vibrational effects are another piece of physics intrinsic to the molecular system. The signature of electron-phonon interactions has been observed in inelastic electron tunneling spectra of SMTs made with these complexes [14]. Moderate coupling of charge and vibrational modes [19] localized to the molecule can strongly both increase $T_{\mathrm{K}}$ and decrease its gate dependence relative to the case with no vibrational coupling [20]. A quantitative estimate of electron-vibrational couplings would facilitate testing this hypothesis, and should be obtainable from further quantum chemistry calculations.

Finally, it is also possible [21] that screening correlations in the mixed valence regime can renormalize the measured $\Gamma$ to a value different than the $\Gamma$ relevant to the Kondo temperature. Full quantum chemistry calculations of molecules bound to realistic Au leads including Kondo and many-body correlations [as done for Co atoms on $\mathrm{Au}(111)[22]]$ are essential to better understanding the observed effects, and are beyond the scope of this Letter.

In measuring the electronic properties of singlemolecule transistors containing transition metal complexes, we observe strong Kondo physics, indicating that conjugated ligands can provide extremely effective coupling of spin degrees of freedom to metal leads. We also find a $T_{\mathrm{K}}\left(V_{G}\right)$ that is vastly weaker than that seen in semiconductor quantum dot realizations of the Kondo effect and expected for the simple model of Fig. 1(a). We have discussed possible explanations for this anomalous dependence in light of electronic structure calculations of the complexes. While a complete understanding will require more sophisticated modeling and further measurements, these data demonstrate that correlated states involving SMTs can exhibit rich effects not seen in their semiconductor quantum dot counterparts.

D. N. acknowledges support from the Research Corporation, the Robert A. Welch Foundation, the David and Lucille Packard Foundation, the Alfred P. Sloan Foundation, and NSF Grant No. DMR-0347253. J.M. T. acknowledges support from DARPA and the ONR. M. R.P. and T. B. acknowledge ONR (Grant No. N000140211046) and DoD CHSSI program. T. B. acknowledges NSF Grant No. NIRT-0304122. The authors thank Dr. M. Fabian and
Professor G. Palmer for EPR characterization, Dr. M.P. Stewart for XPS characterization, and Professor Q. Si for a critical reading of the manuscript.

[1] J. Kondo, Prog. Theor. Phys. 32, 37 (1964).

[2] V. Madhavan, W. Chen, T. Jamneala, M. F. Crommie, and N. S. Wingreen, Science 280, 567 (1998).

[3] K. Nagaoka, T. Jamneala, M. Grobis, and M. F. Crommie, Phys. Rev. Lett. 88, 077205 (2002).

[4] D. Goldhaber-Gordon, H. Shtrikman, D. Mahalu, D. Abusch-Magder, U. Meirav, and M. A. Kastner, Nature (London) 391, 156 (1998).

[5] S. H. Cronenwett, T. H. Oosterkamp, and L. P. Kouwenhoven, Science 281, 540 (1998).

[6] D. Goldhaber-Gordon, J. Göres, M. A. Kastner, H. Shtrikman, D. Mahalu, and U. Meirav, Phys. Rev. Lett. 81, 5225 (1998).

[7] W. G. van der Wiel, S. De Franceschi, T. Fujisawa, J. M. Elzerman, S. Tarucha, and L. P. Kouwenhoven, Science 289, 2105 (2000).

[8] H. Park, J. Park, A. K. L. Lim, E. H. Anderson, A. P. Alivisatos, and P. L. McEuen, Nature (London) 407, 57 (2000).

[9] W. Liang, M.P. Shores, M. Bockrath, J. R. Long, and H. Park, Nature (London) 417, 725 (2002).

[10] J. Park, A. N. Pasupathy, J. I. Goldsmith, C. Chang, Y. Yaish, J. R. Petta, M. Rinkowski, J. P. Sethna, H. D. Abruña, P. L. McEuen, and D. C. Ralph, Nature (London) 417, 722 (2002).

[11] L. H. Yu and D. Natelson, Nano Lett. 4, 79 (2004).

[12] A. N. Pasupathy, R. C. Bialczak, J. Martinek, J. E. Grose, L. A. K. Donev, P. L. McEuen, and D. C. Ralph, Science 306, 86 (2004).

[13] J. W. Ciszek, M. P. Stewart, and J. M. Tour, J. Am. Chem. Soc. 126, 13172 (2004).

[14] L. H. Yu, Z. K. Keane, J. W. Ciszek, L. Cheng, M. P. Stewart, J. M. Tour, and D. Natelson, Phys. Rev. Lett. 93, 266802 (2004).

[15] See EPAPS Document No. E-PRLTAO-95-089552 for a discussion of molecule characterization and density functional calculations. A direct link to this document may be found in the online article's HTML reference section. The document may also be reached via the EPAPS homepage (http://www.aip.org/pubservs/epaps.html) or from ftp.aip. org in the directory /epaps/. See the EPAPS homepage for more information.

[16] H. Park, A. K. L. Lim, J. Park, A. P. Alivisatos, and P. L. McEuen, Appl. Phys. Lett. 75, 301 (1999).

[17] F. D. M. Haldane, Phys. Rev. Lett. 40, 416 (1978).

[18] J. Bonča and J.E. Gubernatis, Phys. Rev. B 47, 13137 (1993).

[19] P. S. Cornaglia, H. Ness, and D. R. Grempel, Phys. Rev. Lett. 93, 147201 (2004); P. S. Cornaglia, D. R. Grempel, and H. Ness, Phys. Rev. B 71, 075320 (2005).

[20] D. R. Grempel (private communication).

[21] Q. Si and G. Kotliar, Phys. Rev. B 48, 13881 (1993).

[22] O. Újsághy, J. Kroha, L. Szunyogh, and A. Zawadowski, Phys. Rev. Lett. 85, 2557 (2000). 